\title{
Recent Results of Solid-State Spectroscopy
}

\section{Cornelia Jäger ${ }^{2}$, Thomas Posch ${ }^{3}$, Harald Mutschke ${ }^{1}$, Simon Zeidler ${ }^{1}$, Akemi Tamanai ${ }^{4}$ and Bernard L. de Vries $^{5}$}

${ }^{1}$ Astrophysikalisches Institut, Friedrich-Schiller-Universität Jena, Schillergässchen 2-3, D-07745 Jena, Germany

${ }^{2}$ Laboratory Astrophysics and Cluster Physics Group, Max Planck Institute for Astronomy and Institute of Solid State Physics, Friedrich-Schiller-Universität Jena, Max-Wien-Platz 1,

D-07745 Jena, Germany, Email: cornelia.jaeger@uni-jena.de

${ }^{3}$ Institut für Astronomie, Türkenschanzstraße 17, A-1180 Vienna, Austria

${ }^{4}$ Kirchhoff-Institut für Physik, Universität Heidelberg, Im Neuenheimer Feld 227, D-69120

Heidelberg, Germany

${ }^{5}$ Instituut voor Sterrenkunde, Katholieke Universiteit Leuven, Celestijnenlaan 200D, 3001

Leuven, Belgium

\begin{abstract}
Solid state spectroscopy continues to be an important source of information on the mineralogical composition and physical properties of dust grains both in space and on planetary surfaces. With only a few exceptions, artificially produced or natural terrestrial analog materials, rather than 'real' cosmic dust grains, are the subject of solid state astrophysics. The Jena laboratory has provided a large number of data sets characterizing the UV, optical and infrared properties of such cosmic dust analogs. The present paper highlights recent developments and results achieved in this context, focussing on 'non-standard conditions' such as very low temperatures, very high temperatures and very long wavelengths.
\end{abstract}

Keywords. methods: laboratory; ISM: dust, extinction; stars: AGB and post-AGB; circumstellar matter

\section{Introduction}

Solids are strangers in space. By far the largest part of the baryonic matter in the universe is in the gas or plasma state. Less than $1 \%$ of cosmic matter consists of solids, such as ices, dust grains or terrestrial planets. Yet, many of the molecules known from astrochemical investigations would not have formed without the catalytic role that the surfaces of solids play in various environments (e.g. in circumstellar shells, in the interstellar medium, protoplanetary disks, etc.).

Proper understanding of solids in space requires studies on analog materials in terrestrial laboratories. Here we shall focus on the infrared spectra of cosmic dust analogs (CDAs), which are a small, but crucial subset of CDA properties. An obvious goal of infrared spectroscopy of CDAs is to create databases of dielectric functions enabling us to identify cosmic dust species from their observed astronomical spectra. One such database is the Jena Database of Optical Constants of Solids:

http://www .astro.uni-jena.de/Laboratory/Database/databases.html.

For most of the solids discussed here, data files of their optical constants can be retrieved from this website. It should be noted that while a precise analytical characterization has been performed for all materials that are present in the Jena Database, the respective detailed results of physico-chemical analyses are not contained there, but only in the pertinent scientific articles. 
Our paper is structured as follows. Section 2 is on mid-infrared spectroscopy of solids, while section 3 is on far-infrared spectroscopy. In both sections, we focus on recent measurements, a substantial part of which were performed at either cryogenic temperatures (especially in the case of the FIR-measurements) or at high temperatures, namely in order to explore conditions under which cosmic dust doubtlessly exists. At some points, a comparison to astronomical infrared spectra is made, as a part of the above mentioned, still ongoing effort to identify the main components of circumstellar, interstellar and in protoplanetary dust.

\section{Mid-Infrared Spectroscopy}

\subsection{General considerations}

As for the identification of cosmic dust species on the basis of their vibrational bands, the mid-infrared region $(5-50 \mu \mathrm{m})$ turned out to be the most fruitful wavelength range.

For some species of solids - e.g. crystalline silicates and highly ionic crystalline oxides in which intense dipole oscillations can be excited - MIR powder spectra are strongly influenced by the shapes, sizes, and possible agglomeration of the respective particles (e.g. Salisbury et al. 1992). Crystalline grains which are not small compared to the examined wavelengths, as well as powders containing grains strongly deviating from spherical symmetry, show markedly different spectra (band positions, band profiles) than very small spherical grains. For amorphous solids, the influence of the particle shapes on the infrared (powder) spectra is less pronounced.

There are several ways to overcome or 'control' the grain-shape-dependence of infrared spectra. One method is to determine optical constants, $n$ and $k-$ e.g. from reflectance measurements of polished surfaces of bulk material - and to use these quantities for the calculation of small particle spectra for arbitrary grain shapes (e.g. Bohren \& Huffman 1983). Compared to mineral powder spectra - for which the actual grain shapes are often unknown or difficult to disentangle -, calculated small particle spectra are better suited for a comparison with the spectra of cosmic dust, since the grain shape can then be varied in the calculations in a controlled way. Another method to get grip on grain-shapedependence is aerosol spectroscopy; exemplary results are presented in the following subsection.

\subsection{Effects of grain shapes on band profiles examined by aerosol spectroscopy}

The Jena laboratory astrophysics group has adopted the aerosol technique and performed IR spectroscopic measurements in a matrix-free environment (Tamanai et al. 2006, 2009a, 2009b). With this method, it is possible to directly study the influence of particle morphologies on IR spectra. Any spectral influence of a matrix material used in powder spectroscopy (e.g. potassium bromide, $\mathrm{KBr}$ ) is ruled out in aerosol spectroscopy, simply because there is no matrix substance in this case (the particles are free floating).

Images of two forsterite $\left(\mathrm{Mg}_{2} \mathrm{SiO}_{4}\right)$ samples with different shapes obtained by a scanning electron microscope (SEM) are shown in Fig. 1. The image of 'Forsterite a' (left) is dominated by roundish grains. On the other hand, 'Forsterite b' particles (right) are irregularly shaped and show sharp edges. X-ray diffraction has been used to examine the crystal structure of both samples, which turned out to be identical. As shown in Fig. 2, the band profiles measured for forsterite particles with ellipsoidal (roundish) shapes clearly differ from those with shapes including sharp edges. The peaks of the resonances undergo a blueshift of up to $0.22 \mu \mathrm{m}$ for the rounded grains (Tamanai et al. 2006). Although it is difficult to demonstrate that the peak shift is purely caused by the grain shape, a blueshift of the rounded grains is expected, since the resonances of a sphere 


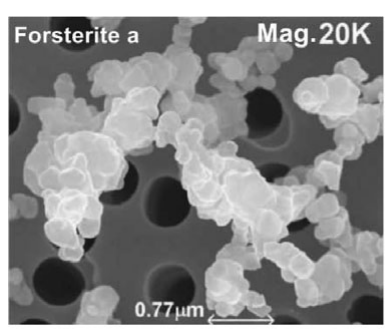

(a)

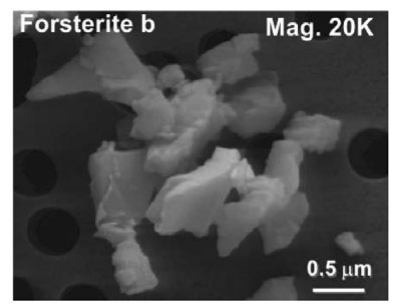

(b)

Figure 1: SEM images of forsterite particles. (a) 'Forsterite a', with nearly spheroidal and ellipsoidal (rounded) grain shapes. (b) Irregular shapes with sharp edged particles dominating in 'Forsterite b'. Note: the black dots are the holes of the polyester membrane filter which have a diameter of $0.5 \mu \mathrm{m}$.

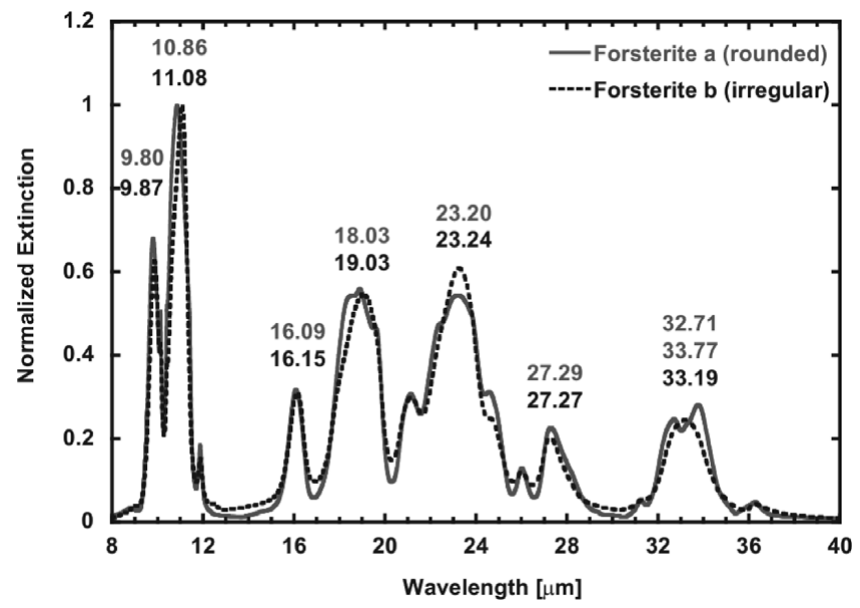

Figure 2: Normalized extinction vs. wavelength for two different shapes of forsterite particles. The grey solid line refers to the 'Forsterite a' (rounded), while the black dotted line refers to the 'Forsterite b' (irregular shapes).

arise at shorter wavelengths, which can be predicted by means of theoretical simulations (Bohren \& Huffman 1983). It can be predicted that resonances of particles far from a spherical shape, such as needle-like ones, are located predominantly at the transverse optical (TO) frequency, which is located at longer wavelengths than the resonance of spherical grains (see also Koike et al. 2010). Another interesting difference between the IR band profiles of rounded and of irregular shaped particles is that rounded grains may produce double peaked (rectangular) profiles (see e.g. the peaks at 19, 23, $33 \mu \mathrm{m}$ ). This tendency has been observed in oxide particle investigations as well (see more details in Tamanai et al. 2009a, b).

The particle size and agglomeration state may partially affect the spectral features as well. Thus it is essential to do theoretical modeling in order to distinguish grain shape effects from grain size and agglomeration effects.

\subsection{High temperature measurements of spinel and corundum and comparison with the observed 'astronomical' $13 \mu \mathrm{m}$ band}

A second 'hot topic' in solid-state astrophysics is to fully cover the range of temperatures that dust grains reach in space. In the case of circumstellar dust, this may imply the necessity to heat the solid samples up to $1000 \mathrm{~K}$. 
Two different cases of high temperature measurements have to be distinguished:

- Annealing: In this case a sample undergoes some persistent structural transition upon heating and keeping it hot for a certain time. The effect of the respective structural change on the dielectric properties, crystal structure etc. can be measured after cooling the sample to room temperature.

- 'In situ' high-T-measurements: These have to be applied if reversible changes (of infrared properties etc.) are expected to occur for a cosmic dust analog, such that for instance its IR reflectance continuously changes upon heating. IR spectra consequently have to be taken while the sample is still hot, which may require sophisticated experimental setups.

While annealing experiments have been carried out for cosmic dust analogs in a number of laboratories, in situ high-T-spectra are so far available for a few substances only.

We performed in situ high-T measurements of the IR properties of corundum $\left(\alpha-\mathrm{Al}_{2} \mathrm{O}_{3}\right)$ and spinel $\left(\mathrm{MgAl}_{2} \mathrm{O}_{4}\right)$, since these dust species are indeed expected to condense at high temperatures (above $1000 \mathrm{~K}$ ) in stellar envelopes (see Gail 2010) and protoplanetary disks.

Both mineral species have been discussed as potential carriers of the so-called $13 \mu \mathrm{m}$ band - a noteworthy infrared feature detected in oxygen-rich AGB stars with low mass loss rates (Posch et al. 1999, Fabian et al. 2001, Sloan et al. 2003). Our measurements set new constraints on the carrier substance of this band and on its possible range of temperatures, as will be discussed below.

For our laboratory measurements, a high-temperature high-pressure (HTHP) cell was used. This water-cooled cell allowed us to carry out the temperature-dependent measurements up to $1073 \mathrm{~K}$. The HTHP cell (type Specac P/N 5850) was integrated into a Bruker 113v Fourier-Transform-Infrared spectrometer. We measured reflection spectra of crystallographically oriented minerals heated to 573,773 , and $973 \mathrm{~K}$, relative to the reflectance of a gold mirror (kept at room temperature). From the reflectance spectra, the complex refractive indices of the minerals have been calculated using a Lorentz oscillator model. In the case of spinel, we used a Lorentz model with five oscillators, while for corundum, 11 and 9 oscillators for the crystallographic orientations $\mathrm{E} \| \mathrm{c}$ and $\mathrm{E} \perp \mathrm{c}$ were needed, respectively. With increasing temperature, the oscillator

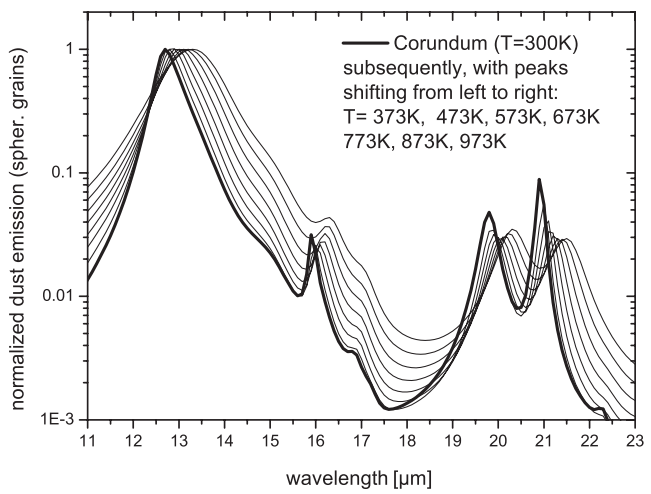

(a)

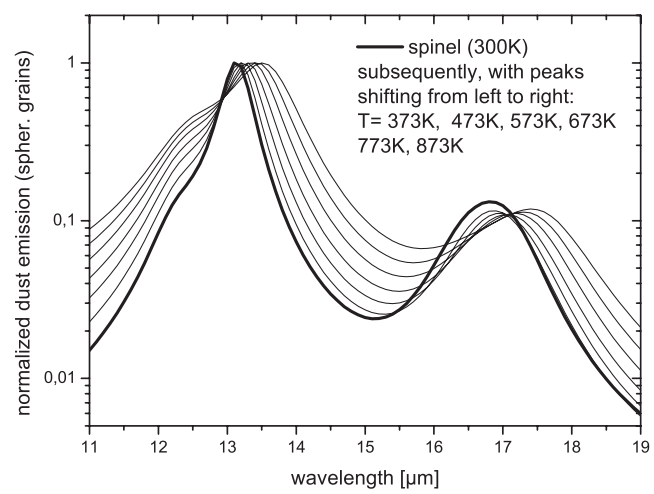

(b)

Figure 3: Normalized emission spectra of small spherical corundum (a) and spinel grains (b) for different temperatures ranging from 300 to $973 \mathrm{~K}$. Orientational averaging has been performed as required by the anisotropy of $\alpha-\mathrm{Al}_{2} \mathrm{O}_{3}$. Note the shift of the peaks to longer wavelengths and the increasing bandwidth as the temperature increases. 


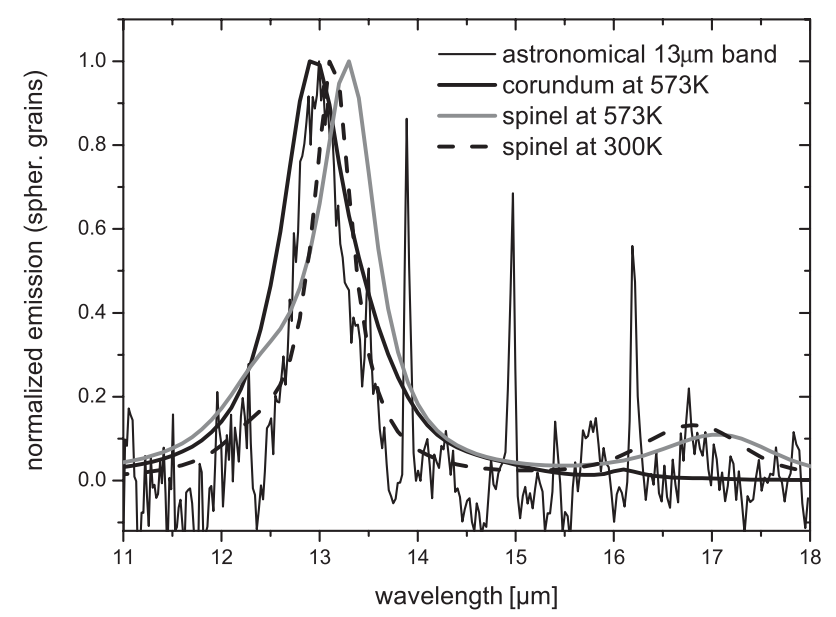

Figure 4: Comparison between the average profile of the astronomical $13 \mu \mathrm{m}$ emission band - as derived by Fabian et al. (2001) for a sample of oxygen-rich AGB stars - with the normalized emission spectra of corundum and spinel grains at temperatures of $300 \mathrm{~K}$ and $573 \mathrm{~K}$.

frequencies generally shift to longer wavelengths and the damping parameters increase strongly, while the oscillator strength changes only slightly. This behavior is expected for resonances in an anharmonic vibrational potential (e.g. Henning \& Mutschke 1997).

In order to derive the complex refractive indices $(n+\mathrm{i} k)$ - also for intermediate temperatures - we fitted the temperature dependence of the oscillator parameters by second order polynomials. In consequence, we obtained analytical expressions for the temperaturedependent optical constants of the measured mineral for each crystallographic axis.

From the optical constants, small-particle spectra $Q(\lambda)$ were calculated for the simplest case of spherical grains in the Rayleigh size limit (particle size $a \ll \lambda$ ). The emitted flux at a given temperature $\mathrm{T}$ finally results from multiplying $Q(\lambda)$ with the corresponding Tdependent blackbody function $B_{\nu}(T)$. The respective emission spectra for temperatures ranging from $300 \mathrm{~K}$ to $973 \mathrm{~K}$ are shown in Fig. 3.

In Fig. 4, we compare the small-spherical-grain emission spectra of spinel and corundum to the average profile of the above mentioned $13 \mu \mathrm{m}$ band as derived by Fabian et al. (2001).

The comparison shows that

i) both spinel and corundum grains - if mainly radiating at a 'suitable' narrow range of temperatures - can account for the average profile of the $13 \mu \mathrm{m}$ band: spinel at about $300 \mathrm{~K}$ and corundum at about $573 \mathrm{~K}$;

ii) at high grain temperatures, the resulting integrated emission both of spinel and of corundum grains becomes too broad as to fit the observed astronomical band (see esp. the spinel spectrum for $573 \mathrm{~K}$; cf. Fig. 3a for corundum at $\mathrm{T}>600 \mathrm{~K}$ ).

As for corundum, it should be noted that only our high temperature measurements enable a successful fitting to the $13 \mu \mathrm{m}$ band. However, the problem remains that corundum has additional weaker bands around $16 \mu \mathrm{m}$ and $20-21 \mu \mathrm{m}$ which have no clear counterparts in the observed astronomical spectra.

\subsection{Spectral properties of carbonaceous material}

We now turn to the spectral properties of carbonaceous materials, another major component of cosmic dust, and start this subsection with a brief introduction into the secrets of 
carbonaceous structures prepared by gas-phase condensation and with a short overview on possible formation processes of carbonaceous solids in astrophysical environments. The structure of particulate carbon materials can be characterized on different length scales describing the short-, medium-, and long-range order of the material. The long-range order describes the size and shape of the primary particles and their agglomeration state. The medium-range order characterizes the arrangement, shape and size of the structural subunits and can be derived by analytical characterization such as high-resolution transmission electron microscopy. The hybridization state of carbon, the nature of the bonds between carbon atoms as well as the incorporation of hydrogen or heteroatoms in the carbon grains can be specified by the short-range order of the material (Schnaiter et al. 1998, Jäger et al. 1999). The short- and medium-range order of carbon grains can be well described by the terms 'amorphous carbon' (AC) or 'hydrogenated amorphous carbon' (HAC). HACs are frequently used to describe the carbonaceous dust component in astrophysical studies (Jones et al. 1990, Duley 1994, Pendleton \& Allamandola 2002, Dartois 2011). In AC and HAC, the carbonaceous structure can be characterized by aromatic islands of different sizes, which are linked by aliphatic structures including $\mathrm{sp}, \mathrm{sp}^{2}$, and $\mathrm{sp}^{3}$ carbon atoms. In the astrophysical literature, the term HAC is preferentially employed for structures containing aromatic units of 2-8 rings (Jones et al. 1990).

In recent laboratory studies on the gas-phase condensation of carbonaceous material, a low- and a high-temperature formation process has been found (Jäger et al. 2009). At condensation temperatures higher than $3500 \mathrm{~K}$, fullerene fragments or complete fullerenes build up the nucleating particles. Fullerene-like carbon grains and fullerene compounds are formed. Low-temperature condensation $(\mathrm{T} \leqslant 1700 \mathrm{~K})$ favors a nucleation and growth process with PAHs as precursors and particle-forming elements resulting in carbonaceous grains revealing well developed planar or slightly bent graphene layers in their interior and a mixture of PAHs as side products.

The MIR spectra of gas-phase condensed carbonaceous solids that may consist either of a mixture of grains and soluble components (PAHs or fullerenes) or of carbonaceous grains exclusively, without a soluble component, show aromatic IR bands (AIBs) as well as aliphatic IR band, partly superimposed on broad plateau features around 8 and $12 \mu \mathrm{m}$ (see Fig. 5). Aliphatic $-\mathrm{CH}_{x}$ absorptions can be observed at $3.4,6.8$, and $7.25 \mu \mathrm{m}$. In fullerene-like carbonaceous grains, saturated aliphatic $-\mathrm{CH}_{x}$ groups are mainly responsible for the links between the fullerene fragments (Jäger et al. 2008). In this material, signatures related to the presence of $-\mathrm{C} \equiv \mathrm{C}$ - triple bonds can be observed in the in-situ recorded IR spectra at 3.0 and $4.7 \mu \mathrm{m}$, which points to the presence of polyynes. For the formation of fullerene-like soot grains, polyynes are discussed as intermediates. They finally form up the fullerene snatches that are incorporated into the grains. Additional bands due to $-\mathrm{C}-\mathrm{C}-$ stretching, $-\mathrm{C}-\mathrm{H}$, and $-\mathrm{C}-\mathrm{C}-$ twisting, wagging, and rocking bands can be expected in the range between 7.4 and $10 \mu \mathrm{m}$. In the $11-15 \mu \mathrm{m}$ range, out-of-plane $-\mathrm{C}-\mathrm{H}$ bending bands can be observed caused by $-\mathrm{C}-\mathrm{H}$ groups at the periphery of the fullerene snatches.

The more aromatic carbonaceous material produced by low-temperature condensation of hydrocarbons in laser pyrolysis (Jäger et al. 2009) show a series of aromatic bands including $3.3,6.3,8.6,11.3,12.3$, and $13.3 \mu \mathrm{m}$ as well as aliphatic IR bands at $3.4,6.8$, and $7.25 \mu \mathrm{m}$ ( $-\mathrm{C}-\mathrm{H}$ stretching and deformation bands). IR features observed beyond $6.7 \mu \mathrm{m}(6.8,7.25,7.93,8.47,9.28,9.72,10.5 \mu \mathrm{m})$ mainly arise from $-\mathrm{C}-\mathrm{C}$ - stretching, $\mathrm{C}-\mathrm{H}$ deformation, and other bands due to $-\mathrm{C}-\mathrm{H}$ twisting, wagging, rocking, and $-\mathrm{C}-$ $\mathrm{C}$ - deformation bands. In this spectral range, a firm assignment of the IR features to a specific functional group is rather difficult even for carbonaceous grains without adsorbed 
PAHs, which may contain a lot of different functional groups with single and double bonds integrated in varying chemical environments leading to a distribution of vibrational bands in this spectral range.

Bands of more diagnostic relevance can be found at wavelengths larger than $11 \mu \mathrm{m}$, which can be assigned to aromatic $=\mathrm{C}-\mathrm{H}$ out-of-plane bending vibrations (see Fig. 5). The $11.3 \mu \mathrm{m}$ feature can be assigned to a single $=\mathrm{C}-\mathrm{H}$ band, whereas the band at around $12.3 \mu \mathrm{m}$ is caused by $2-3$ and the feature at $13.27 \mu \mathrm{m}$ by $3-4$ adjacent $\mathrm{H}$ atoms in the periphery of PAH molecules. The 13.56 and $14.3 \mu \mathrm{m}$ bands can be attributed to around 4 adjacent $\mathrm{H}$ atoms bound to the aromatic ring. The observed IR features in carbonaceous condensates are typical for both non-substituted PAHs or PAHs containing methyl or methylene groups, as well as for carbonaceous grains containing aromatic and aliphatic structures.

Sometimes, also oxygen-containing functional groups such as $-\mathrm{C}=\mathrm{O}$ (carbonyl group) are incorporated into the carbonaceous structures which can be identified at wavelengths between $5.8-6.0 \mu \mathrm{m}$. In addition, higher fullerenes can show vibrational modes in this range (Mordkovich 2000).

Carbonaceous condensates consisting of grain/PAH blends show spectral characteristics that are comparable to the observed IR spectra of post AGB stars and protoplanetary nebulae (Kwok et al. 2001, Hony et al. 2003, Hrivnak et al. 2007). A comparison shown in Fig. 5 demonstrates that nearly all of the IR bands observed in the spectrum of the post-AGB-star HD 56126 are also present in the spectrum of the laboratory lowtemperature condensate. Differences in band ratios, apparent for the $=\mathrm{C}-\mathrm{H}$ out-of-plane bands between 11 and $14 \mu \mathrm{m}$, are due to a higher abundance of small PAHs, or larger PAHs containing outer rings with up to 3 to $4 \mathrm{H}$ atoms compared to larger, compactly condensed species which are thought to be very stable under astrophysical conditions. Carbonaceous particles built up by large PAHs in the growth process and purified from the adsorbed, smaller PAHs by extraction show a better coincidence with the observed bands (see gray solid curve in Fig. 5). However, in order to obtain a dominance of the $11.3 \mu \mathrm{m}$ out-of-plane band due to solo $=\mathrm{C}-\mathrm{H}$ frequently observed in PPNs and PNs, very large $\left(\mathrm{N}_{C} \geqslant 100\right)$ and rather elongated PAH molecules are required (Hony et al. 2001). The comparison reveals that the low-temperature condensate is a promising dust analog for carbonaceous materials produced in carbon-rich AGB stars.

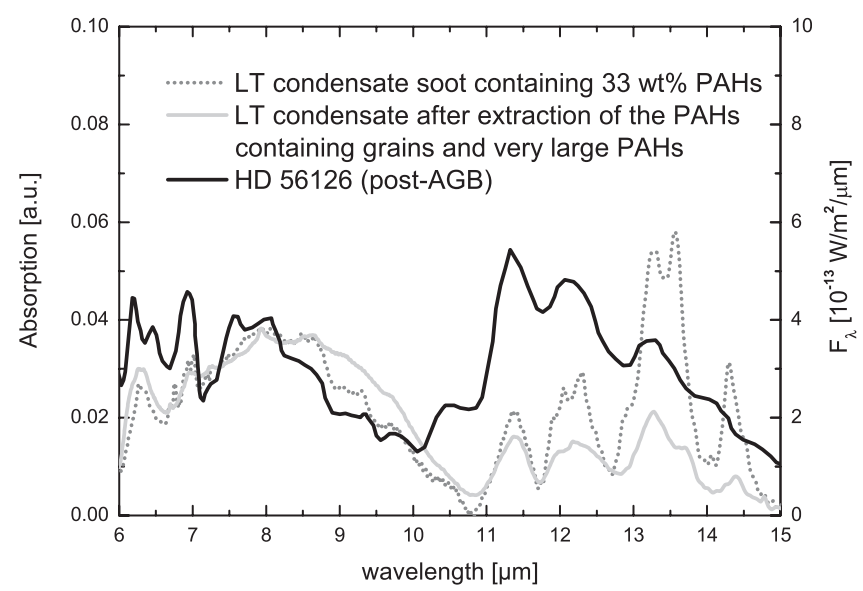

Figure 5: Comparison between the IR spectral properties of a carbonaceous condensate that consists of a mixture of solid carbon particles and PAHs and the observed spectrum of a post AGB star adapted from Hony et al. (2003). 


\section{Far-Infrared Spectroscopy}

\subsection{Selected FIR bands of solids: carbonates, phyllosilicates and the 69 micron forsterite band}

A number of crystalline dielectric solids show phonon bands in the far-infrared range $(\lambda>50 \mu \mathrm{m})$, where the relatively cold dust located at larger distances from stars (e.g. in planetary nebulae or protoplanetary systems) is emitting thermal radiation. If observed, these bands would be of high diagnostic value to assess the mineralogy of this dust and to draw conclusions about its history. The profiles of these bands have been demonstrated to be quite sensitive to temperature, i.e. the bands get sharper and shift to shorter wavelengths at low temperatures. Consequently, it is necessary to perform laboratory spectroscopy at these low temperatures for providing comparison spectra, or for the determination of low-temperature optical constants as input for calculations, respectively (see Sect. 2.2 for the analogous case of high temperatures). Such low-temperature spectroscopic data have been produced by laboratory work in the past years. E.g., temperaturedependent optical constants have been measured for forsterite (Suto et al. 2006) and for carbonates (Posch et al. 2007). These optical constants are very valuable because of their flexibility and independence from the measurement conditions (see Sect. 2.1). Other studies have determined the changes of band positions and band widths with temperature in absorption measurements based on the pellet technique. E.g., the long-wavelength bands of several crystalline silicates have already been measured at low temperatures about ten years ago (Bowey \& Adamson 2002, Chihara et al. 2002).

A more complete investigation of the low-temperature absorption spectra of olivine minerals with different iron contents was presented by Koike et al. (2006). The quantification of the nonlinear peak shift of the $69 \mu \mathrm{m}$ band and of the band broadening with temperature allows to use these bands as thermometers under certain conditions such as at known iron content of the silicate. As an example of how temperature dependent optical constants can be used, Fig. 6 shows the $69 \mu \mathrm{m}$ band in the Herschel/PACS spectrum of the post-AGB star HD161796 (obtained by the MESS consortium, Groenewegen et al. 2011; see also de Vries et al. 2011). A one-temperature fit of the $69 \mu \mathrm{m}$ band indicates that the forsterite grains in this system have a temperature of the order of $124 \mathrm{~K}$. For our derivation of the temperature, we used the laboratory measurements of forsterite's optical constants at different temperatures by Suto et al. (2006).

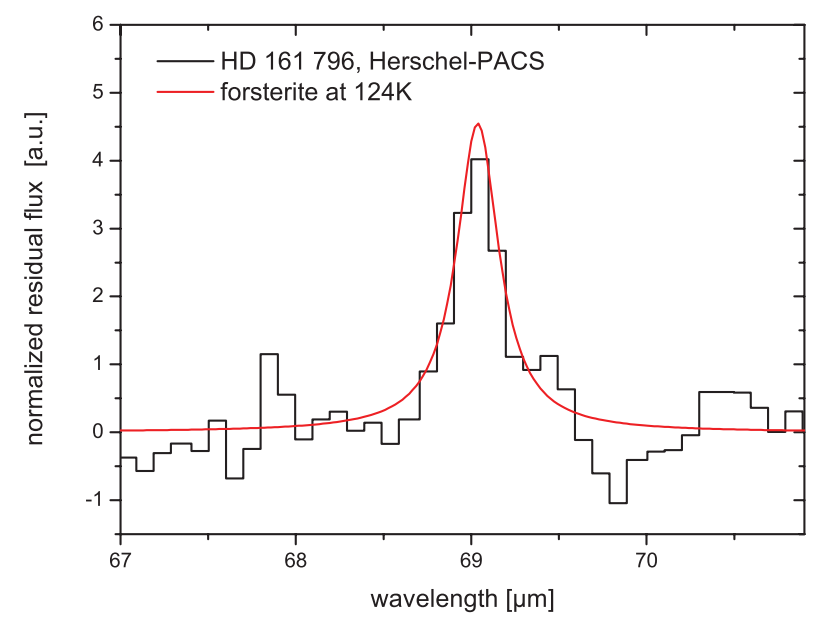

Figure 6: The $69 \mu \mathrm{m}$ band in the (binned) Herschel-PACS spectrum of the post-AGB star HD161796. The 'smooth' line shows the best fit, obtained with forsterite at $\mathrm{T}=124 \mathrm{~K}$. 
Apart from olivines, several other crystalline silicates show clear FIR bands in the long-wavelength range as well. In Fig. 7 , bands of diopside $\left(\mathrm{CaMgSiO}_{3}\right)$ and of the hydrous silicates serpentine $\left((\mathrm{Mg}, \mathrm{Fe})_{3} \mathrm{Si}_{2} \mathrm{O}_{5}(\mathrm{OH})_{4}\right)$ and talc $\left(\mathrm{Mg}_{3}\left[\mathrm{Si}_{4} \mathrm{O}_{10}(\mathrm{OH})_{2}\right]\right)$ are shown for example. Diopside has a very strong $66 \mu \mathrm{m}$ band (note the five-times reduction of the peak intensity in our plot). It is an abundant constituent of early condensates in the solar nebula (see, e.g. Posch et al. 2007a), but the band has not yet been identified in the spectra of protoplanetary disks. By contrast, it may in fact contribute to a band seen at this wavelength in planetary nebulae (Kemper et al. 2002, Chihara et al. 2007).

Hydrous silicates are weathering products of silicate minerals including olivines and pyroxenes and they are abundant in some classes of primitive meteorites. Hydrous silicates were also discussed to be a carrier of a broad $100 \mu \mathrm{m}$ emission band detected in Herbig Ae/Be stars (Malfait et al. 1999). Far-infrared absorption spectra of hydrous silicates have been measured by different laboratory astrophysics groups (Koike et al. 1982, Koike and Shibai 1990, Hofmeister and Bowey 2006, Mutschke et al. 2008). The spectra show very interesting features such as the longest-wavelength phonon bands known to our knowledge (e.g. one at about $270 \mu \mathrm{m}$ for minerals of the chlorite group). However, far-infrared emission features could not be successfully assigned to bands of hydrous silicates so far (see Fig. 8).

Finally, broad far-infrared bands observed in the emission spectrum of the planetary nebula NGC 6302 have been proposed to be caused by carbonate dust (Kemper et al. 2002). Carbonates have extremely strong far-infrared bands (note the ten-times reduction of the peak intensity in Fig. 7), so that already small amounts of these minerals, which may be formed by non-equilibrium reactions of silicates with a $\mathrm{H}_{2} \mathrm{O}-\mathrm{CO}_{2}$-rich gas (Toppani et al. 2005), should be detectable. Low-temperature measurements of the optical constants of carbonates by Posch et al. (2007) largely confirmed this possible assignment for NGC 6302, but did not give support to an assignment to a $100 \mu \mathrm{m}$ emission feature observed in protostars (Chiavassa et al. 2005).

\subsection{Dust continuum opacities in the submillimeter wavelength range}

\subsubsection{Wavelength and temperature dependence}

Apart from the far-infrared phonon bands, which may provide information about the composition and temperature of cold, mostly crystalline dust, the underlying continuum

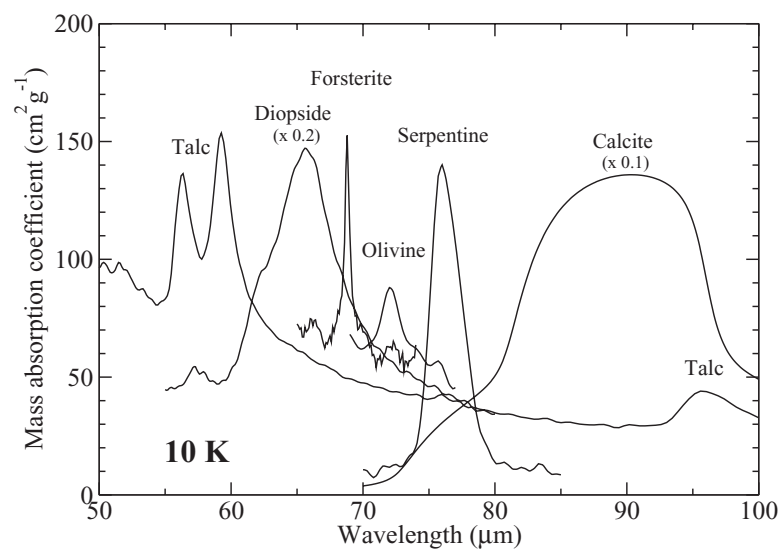

Figure 7: Far-infrared bands of a variety of minerals measured at a temperature of $10 \mathrm{~K}$. The calcite spectrum has been calculated from the optical constants by using the CDE model, whereas all other spectra have been measured on submicron-sized particles embedded in polyethylene pellets. 


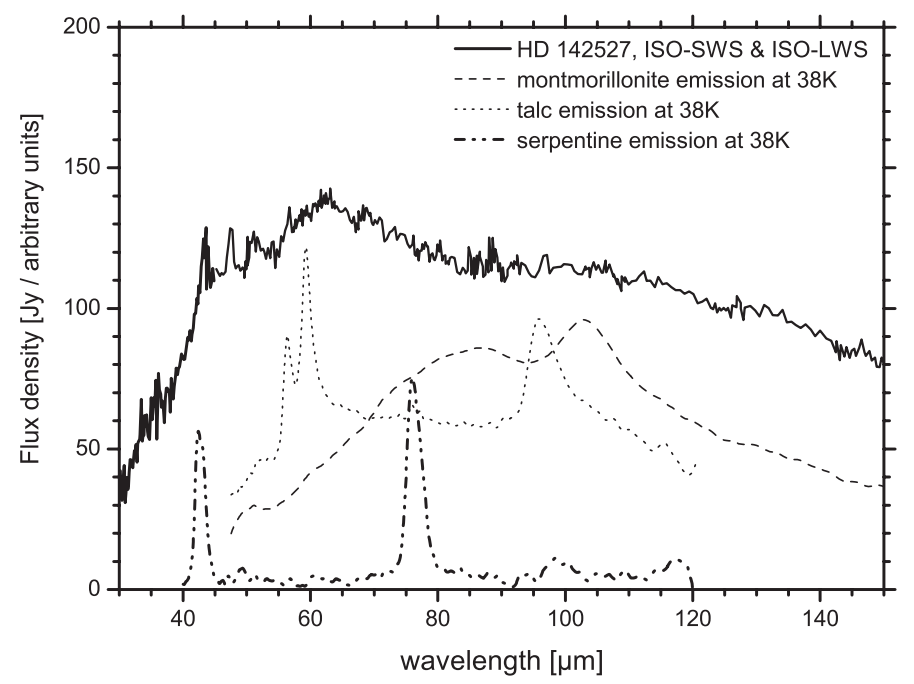

Figure 8: Comparison of simulated emission of cold hydrous silicate dust with the thermal emission of the Herbig AeBe object HD 142527 (after Mutschke et al. 2008).

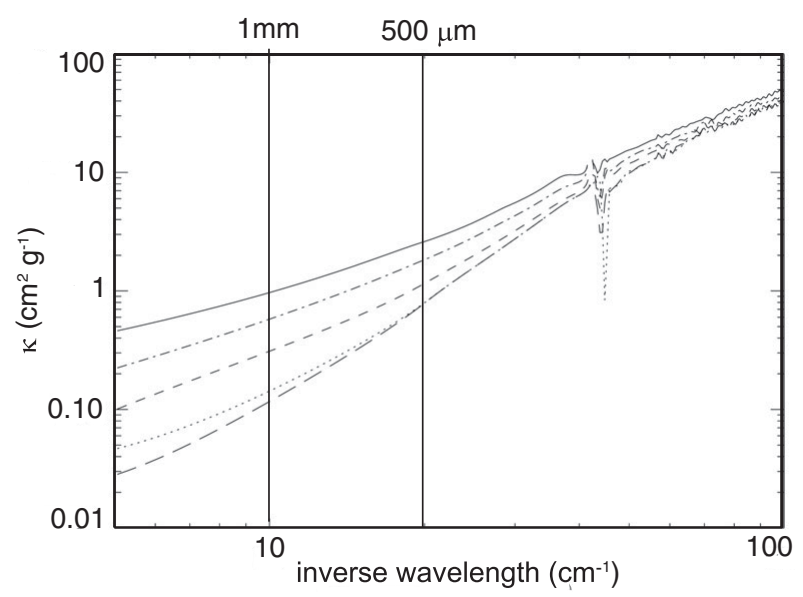

Figure 9: Mass absorption coefficient for MgSiO3, synthetized by sol-gel reaction at $300 \mathrm{~K}$ (solid line), $200 \mathrm{~K}$ (dot-dashed line), $100 \mathrm{~K}$ (short dashed line), $30 \mathrm{~K}$ (dotted line), and $10 \mathrm{~K}$ (long-dashed line) (Boudet et al. 2005).

dust opacity is of great importance. For example, one of the big uncertainties in determining the amount of cold dust in interstellar space arises indeed from the uncertainty in the emissivity of the dust particles at these wavelengths. Furthermore, models of the spatial dust distribution of circumstellar dust, e.g. in planetary systems (debris disks) depend on the wavelength and temperature dependence of the dust opacity.

In contrast to the far-infrared bands, the continuum opacity of dust in most astrophysical environments is determined by that of amorphous solids, especially amorphous silicates, because their emissivity is generally higher at far-infrared wavelengths. The reason for that is the possibility of additional low-energetic transitions due to structural disorder. 
Table 1: Continuum opacities of dust species at low temperatures (4-24 K). The values are given as mass absorption coefficients at $100 \mu \mathrm{m}$ (read from figures - approximate) and $1 \mathrm{~mm}$ wavelength, plus the power-law slope value $\beta$ at $1 \mathrm{~mm}$ wavelength (for forsterite, we refer to the slope at $40-80 \mu \mathrm{m})$. Room-temperature values are given in parentheses.

\begin{tabular}{|c|c|c|c|c|}
\hline Dust species & 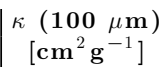 & $\begin{array}{c}\kappa(\mathbf{1} \mathbf{m m}) \\
{\left[\mathbf{c m}^{2} \mathbf{g}^{-1}\right]}\end{array}$ & $\beta$ & Reference \\
\hline Am. Fe-silicate ("FAYA") & $95(110)$ & $0.86(5.0)$ & $2.04(1.35)$ & Mennella et al. (1998) \\
\hline Am. Mg-Silicate $\left(\mathrm{MgSiO}_{3}\right.$ glass $)$ & $45(50)$ & $0.22(0.75)$ & $2.14(1.58)$ & Boudet et al. (2005) \\
\hline Am. Mg-Silicate $\left(\mathrm{MgSiO}_{3}\right.$ sol-gel $)$ & $40(50)$ & $0.12(0.98)$ & $2.74(1.44)$ & Boudet et al. (2005) \\
\hline Am. Mg-Silicate $\left(\mathrm{MgSiO}_{3}\right.$ sol-gel $)$ & & 0.28 & $\sim 2.0$ & Agladze et al. (1996) \\
\hline "Astronomical silicate" (spheres) & 29 & 0.30 & 1.6 & Li \& Draine (2001) \\
\hline Forsterite & $2.1(20)$ & & $5.0(2.6)$ & Chihara et al. (2001) \\
\hline Forsterite & $80(120)$ & $0.16(0.43)$ & $2.32(2.04)$ & Mennella et al. (1998) \\
\hline Forsterite (unheated) & & 0.22 & & Agladze et al. (1996) \\
\hline Am. Carbon ("BE") & $300(500)$ & $21(72)$ & $1.15(0.76)$ & Mennella et al. (1998) \\
\hline
\end{tabular}

Among them, the disorder-induced phonon transitions give a temperature-independent contribution which has been shown by Schlömann (1964) to lead to a $\lambda^{-2}$ wavelength dependence at shorter and a $\lambda^{-4}$ dependence at longer wavelengths (see also Meny et al. 2007). A strongly temperature-dependent behavior is, however, introduced by additional low-energetic tunneling transitions of structural groups in the amorphous network.

First studies of the temperature-dependent absorption coefficient of amorphous silicates in the sub-millimeter and millimeter continuum have been performed by Agladze et al. (1996), Henning \& Mutschke (1997) and Mennella et al. (1998). Recently, these studies have been extended to a larger set of amorphous silicate materials measured in a wide wavelengths and temperature range (Boudet et al. 2005, Coupeaud et al. 2010; see also their poster at this conference). In these studies, the power-law spectral index $\beta$ of absorption was found to increase with decreasing temperature, especially at wavelengths larger than about $300 \mu \mathrm{m}$. For amorphous $\mathrm{Mg}$ silicates and $\mathrm{SiO}_{2}$, this anti-correlation of $\mathrm{T}$ and $\beta$ is demonstrated in Fig. 9 (values taken from Boudet et al. 2005). The spectral index varies between 1 and 3 for temperatures between 300 and $10 \mathrm{~K}$, which confirms the expectations from theory.

In Tab. 1 we summarize opacity values and power-law indices for a number of amorphous and crystalline Mg- and Fe-silicates, taken from four of the mentioned publications (apologies for being not complete). Additionally, we compare with values measured for an amorphous carbon by Mennella et al. (1998) and with the numbers calculated for spherical grains composed of the model material "astronomical silicate (astrosil)" (Li \& Draine 2001).

At a wavelength of $1 \mathrm{~mm}$, the low-temperature opacity values of amorphous magnesium silicates seem to concentrateconverge to values of $0.1-0.3 \mathrm{~cm}^{2} \mathrm{~g}^{-1}$, which is close to the "astrosil" value. However, we have to mention that Agladze et al. (1996) have reported higher values up to $3.7 \mathrm{~cm}^{2} \mathrm{~g}^{-1}$ for some of their sol-gel produced silicate samples as well. The reason for that is not clear, but may have to do with a high porosity of these samples. Fe-silicates seem to have somewhat higher opacities, and a somewhat stronger temperature dependence, but this is only based on one measurement so far (Mennella et al. 1998). For mixed $\mathrm{Mg} / \mathrm{Fe}$ silicates, investigations are still lacking. The power-law exponent around $1 \mathrm{~mm}$ wavelength has typically values between 2 and 3 for amorphous silicates. The "astrosil" value has been reduced in order to better match galactic dust emission data. Such values seem to correspond to certain data in the Agladze et al. measurements, but just these have unusually high opacity values at the same time. 
For amorphous carbon, a similar temperature dependence of the opacity as for amorphous silicates has been reported by Mennella et al. (1998), although at a strongly enhanced opacity level (see Tab. 1). The excitation mechanisms behind this could actually be totally different because contributions from free charge carriers might be dominating (see following subsection).

For crystalline silicates, the measured values do not seem to be in perfect agreement so far. For forsterite, Mennella et al. (1998) and Agladze et al. (1996) found low-temperature opacity and $\beta$ values, which were similar to those of the amorphous silicates, although for the same composition $\kappa$ values lower by a factor of four were reported (Agladze et al. 1996) and the temperature dependence was considerably weaker. Chihara et al. (2001), however, measured values at $100 \mu \mathrm{m}$ wavelengths, which were only one order of magnitude higher, and therefore in disagreement with a $\beta \sim 2$ behavior. This discrepancy certainly deserves further investigation.

\subsubsection{Grain shape dependence and carbonaceous materials}

For carbonaceous materials, the FIR-behavior is not only influenced by the internal structure, but also strongly by the morphology of the carbon grains. It is well known that the clustering of carbon grains decreases the spectral index $\beta$ very strongly (Stognienko et al. 1995, Michel et al. 1996). The influence of the internal structure on the far-infrared behavior of differently structured carbon materials was already studied by Koike et al. (1995), Mennella et al. (1998), and Papoular et al. (1996). These measurements were performed in transmission using polyethylene pellets. However, one has to keep in mind that this method cannot disentangle the structural and morphological effects on the FIR absorption.

For the interpretation of observational FIR data provided by ground-based or satelliteborne observations such as with the Herschel satellite, the opacity of small grains in vacuum is required, which can be calculated from the optical constants $n$ and $k$ for spherical shapes by the Mie theory. In addition, shape effects can roughly be simulated by a continuous distribution of ellipsoids with the same probability of all shape parameters (CDE) in the Rayleigh limit (Bohren \& Huffman 1983). In Fig. 10, the calculated

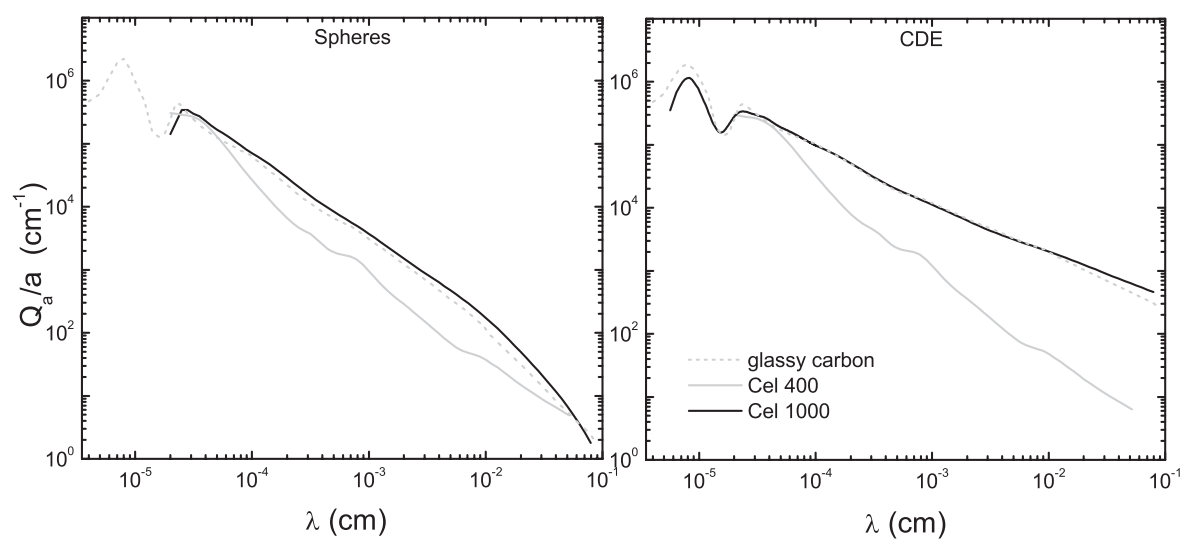

Figure 10: Absorption efficiency divided by particle radius calculated for spherical particles and ellipsoidal grains in vacuum from the optical data of pyrolized cellulose materials. 
normalized absorption efficiencies of two carbon materials ('cel 400' and 'cel 1000') are compared with those of glassy carbon. The two materials represent a $\mathrm{sp}^{3}$ hybridization dominated non-conducting material ('cel 400') and an aromatic carbonaceous material with a high number of free charge carriers ('cel 1000'; see Jäger et al. 1998).

Whereas in case of the aliphatic-dominated carbon material 'cel 400' the spectral in$\operatorname{dex} \beta$ in the FIR region $\left(\lambda \geqslant 100 \mu \mathrm{m}\right.$ or $\left.10^{-2} \mathrm{~cm}\right)$ is about 1.25 irrespective of the particle model, for the aromatic sample carbonized at $1000{ }^{\circ} \mathrm{C}$ it is 2.28 for spherical particles, but only 0.71 for agglomerated (CDE) particles. The latter value is very close to the one measured by Mennella et al. (1998) for amorphous carbon particles embedded in a polyethylene pellet (see Table 1), although the absolute absorption coefficient is in between those calculated for spherical and CDE particles. This may indicate that in the pellet the carbon particles are strongly aggregated. We conclude that for cosmic particles formed of conducting materials such as highly graphitized amorphous carbon, the far-infrared emissivity can be very high, but this will strongly depend on the grain morphology.

\section{References}

Agladze, N. I., Sievers, A. J., Jones, S. A., Burlitch, J. M., \& Beckwith, S. V. W., 1996, ApJ, 462,1026

Begemann, B., Dorschner, J., Henning, Th., et al. 1997, ApJ, 476, 199

Bohren, C. F. \& Huffman, D. R. 1983, Absorption and Scattering of Light by Small Particles (New York: John Wiley)

Boudet, N., Mutschke, H., Nayral, C., Jäger, C., Bernard, J.-P., Henning, Th., \& Meny, C. 2005, ApJ, 633, 272

Bowey, J. E. \& Adamson, A. J., 2002, MNRAS, 334, 94

Chiavassa, A., Ceccarelli, C., Tielens, A. G. G. M., Caux, E., \& Maret, S., 2005, Astron. Astrophys., 432,547

Chihara, H., Koike, C., Tsuchiyama, A., Tachibana, S., \& Sakamoto, D., 2002, Astron. Astrophys., 391, 267

Chihara, H., Koike, C., \& Tsuchiyama, A., 2007, Astron. Astrophys., 464, 229

Coupeaud, A., Demyk, K., Meny, C., \& Nayral, C., 2010, in: SF2A-2010: Proceedings of the Annual meeting of the French Society of Astronomy and Astrophysics, Eds. S. Boissier, M. Heydari-Malayeri, R. Samadi and D. Valls-Gabaud, p. 205

Dartois, E., 2011, EAS Publications Series 46, 381

De Vries, B. L., Klotz, D., Lombaert, R., et al., 2011, in: F. Kerschbaum, Th. Lebzelter, B. Wing (eds.), Why Galaxies Care About AGB Stars II, ASP Conf. Ser., vol. 445, in press

Duley, W., 1994, ApJ, 430, L133

Fabian, D., Jäger, C., Henning, Th., Dorschner, J., \& Mutschke, H. 2000, Astron. Astrophys., 364,282

Fabian, D., Posch, Th., Mutschke, H., Kerschbaum, F., \& Dorschner, J., 2001, Astron. Astrophys., 373,1125

Gail, H.-P., 2010, Formation and Evolution of Minerals in Accretion Disks and Stellar Outflows, in: Th. Henning (ed.), Astromineralogy, 2nd ed., Lecture Notes in Physics (Berlin and Heidelberg: Springer Verlag)

Groenewegen, M. A. T., Waelkens, C., Barlow, M. J., Kerschbaum, F., Garcia-Lario P., et al., 2011, Astron. Astrophys., 526, A162

Henning, Th. \& Mutschke, H. 1997, Astron. Astrophys., 327, 743

Hofmeister, A. M. \& Bowey, J. E., 2006, MNRAS, 367, 577

Hony, S., Van Kerckhoven, C., Peeters, E., Tielens, A. G. G. M., Hudgins, D. M., \& Allamandola, L. J., 2001, Astron. Astrophys., 370, 1030

Hony, S., Tielens, A. G. G. M., Waters, L. B. F. M., \& de Koter, A. 2003, Astron. Astrophys., 402, 211

Hrivnak, B. J., Geballe, T. R., \& Kwok, S. 2007, ApJ, 662, 1059 
Hoppe, P., 2009, ASP Conf. Ser., 414, 149

Jäger, C., Mutschke, H., \& Henning, Th., 1998, Astron. Astrophys., 332, 291

Jäger, C., Henning, Th., Spillecke, N., \& Schlögl, R., 1999, J. Non-Cryst. Solids 258, 161

Jäger, C., Mutschke, H., Henning, Th., \& Huisken, F., 2008, ApJ 689, 249

Jäger, C., Huisken, F., Mutschke, H., Jansa, I. L., \& Henning, Th., 2009, ApJ 696, 706

Jones, A. P., Duley, W. W., \& Williams, D. A., 1990, Q. Jl. R. Astr. Soc. 31, 567

Kemper, F., Jäger, C., Waters, L. B. F. M., Henning, Th., Molster, F. J., Barlow, M. J., Lim, T., \& de Koter, A., 2002, Nature, 415, 295

Koike, C., Hasegawa, H., \& Hattori, T., 1982, Astrophys. Space Sci., 88, 89

Koike, C. \& Shibai, H., 1990, MNRAS, 246, 332

Koike, C., Kimura S., Kaito C., 1995, ApJ 446, 902

Koike, C., Mutschke, H., Suto, H., Naoi, T., Chihara, H., Henning, Th., Jäger, C., Tsuchiyama, A., Dorschner, J., \& Okuda, H., 2006, Astron. Astrophys., 449, 583

Koike, C., Imai, Y., Chihara, H., Suto, H., Murata, K., Tsuchiyama, A., Tachibana, S., \& Ohara, S., 2010, ApJ, 709, 983

Kwok, S., Volk, K., \& Bernath, P. 2001, ApJ, 55, L87

Li, A. \& Draine, B. T., 2001, ApJ, 554, 778

Malfait, K., Waelkens, C., Bouwman, J., de Koter, A., \& Waters, L. B. F. M., 1999, Astron. Astrophys., 345, 181

Mennella, V., Brucato, J., Colangeli, L., Palumbo, P., Rotundi, A., \& Bussoletti, E., 1998, ApJ, 496, 1058

Meny, C., Gromov, V., Boudet, N., Bernard, J.-P., Paradis, D., \& Nayral, C., 2007, Astron. Astrophys., 468, 171

Michel, B., Henning, Th., Stognienko, R., \& Rouleau, F., 1996, ApJ 468, 834

Mordkovich, V. Z., 2000, Chem. Mater. 12, 2813

Mutschke, H., Zeidler, S., Posch, Th., Kerschbaum, F., Baier, A., \& Henning, Th., 2008, Astron. Astrophys., 492, 117

Papoular, R., Conard, J., Guillois, O., Nenner, I., Reynaud, C., Rouzaud, J.-N., 1996 Astron. Astrophys. 315, 222

Pendleton, Y. J. \& Allamandola, L. J., 2002, Astrophys. J. Suppl. Ser. 138, 75

Posch, Th., Kerschbaum, F., \& Mutschke, H., et al., 1999, Astron. Astrophys., 352, 609

Posch, Th., Mutschke, H., Trieloff, M., \& Henning, Th., 2007, ApJ 656, 615

Posch, Th., Baier, A., Mutschke, H., \& Henning, Th., 2007, ApJ, 668, 993

Salisbury, J. W., et al., 1992, Infrared $(2.1-25 \mu \mathrm{m})$ Spectra of Minerals (Baltimore and London: The John Hopkins University Press), p. xv

Schlömann, E., 1964, Phys. Rev., 135, A413

Schnaiter, M., Mutschke, H., Dorschner, J., Henning, Th., \& Salama, F., 1998, Astrophys. J. 498, 486

Sloan, G. C., Kraemer, C., Goebel, J., \& Price, S. 2003, ApJ, 594, 483

Stognienko, R., Henning, Th., \& Ossenkopf, V., 1995, Astron. Astrophys. 296, 797

Suto, H., Sogawa, H., Tachibana, S., Koike, C., Karoji, H., Tsuchiyama, A., Chihara, H., Mizutani, K., Akedo, J., Ogiso, K., Fukui, T., \& Ohara, S., 2006, MNRAS, 370, 1599

Tamanai, A., Mutschke, H., Blum, J., \& Meeus, G., 2006, ApJ, 648, 147

Tamanai, A., Mutschke, H., \& Blum, J., 2009a, Astronomical Society of the Pacific Conference Series, 414, 438

Tamanai, A., Mutschke, H., Blum, J., Posch, Th., Koike, C., \& Ferguson, J. W., 2009b, Astron. Astrophys., 501, 251

Toppani, A., et al., 2005, Nature, 437, 1121

\section{Discussion}

Goumans: You mentioned that if you have the optical constants, one can get the spectrum of grains of any shape and size. What about very small grains, or clusters, that do not behave like bulk material any more? Is spectroscopy on those desirable and possible? 
Posch: Studies of clusters, including infrared spectroscopy, can certainly help to clarify the nature of some unidentified broad infrared bands that were hitherto assigned to mineral species. However, IR spectroscopy of clusters requires very sophisticated techniques and will therefore take even more time than solid state spectroscopy. Additionally, the spectra critically depend on the cluster size, so experiments must be carried out for a wide range of possible cluster sizes. 